\title{
Comparative Proteomic Profile of the Human Umbilical Cord Blood Exosomes between Normal and Preeclampsia Pregnancies with High-Resolution Mass Spectrometry
}

\author{
Ruizhe Jia ${ }^{a}$ Jingyun Lib Can Rui ${ }^{a}$ Hui Jia Hongjuan Ding ${ }^{a}$ Yuanqing Lu ${ }^{a}$ Wei Dec \\ Lizhou Sun $^{\text {d }}$ \\ aDepartment of Obstetrics, Nanjing Maternity and Child Health Care Hospital Affiliated to Nanjing \\ Medical University, Nanjing, 'bState key Laboratory of Reproductive Medicine, Department of \\ Plastic\&Cosmetic Surgery, Nanjing Maternity and Child Health Care Hospital Affiliated to Nanjing \\ Medical University, Nanjing, 'Nanjing Medical University, Nanjing, dDepartment of Obstetrics and \\ Gynecology, the First Affiliated Hospital of Nanjing Medical University, Nanjing, China
}

\section{Key Words}

Exosomes Proteomic profile $\cdot$ Umbilical cord blood Preeclampsia $\cdot$ High-resolution mass spectrometry

\begin{abstract}
Background/Aims: Exosomes are extracellular vesicles that are involved in several biological processes. The roles of proteins from human umbilical cord blood exosomes in the pathogenesis of preeclampsia remains poorly understood. Methods: In this study, we used high-resolution LC-MS/MS technologies to construct a comparative proteomic profiling of human umbilical cord blood exosomes between normal and preeclamptic pregnancies. Results: A total of 221 proteins were detected in human umbilical cord blood exosomes, with 14 upregulated and 15 downregulated proteins were definitively identified between preeclamptic and control pregnancies. Further bioinformatics analysis (Gene ontology and Kyoto Encyclopedia of Genes and Genomes pathway analysis) indicated that these differentially expressed proteins correlate with enzyme regulator activity, binding, extracellular region, cell part, biological regulation, cellular process and complement and coagulation cascades occurring during pathological changes of preeclampsia. Conclusion: Our results show significantly altered expression profiles of proteins in human umbilical cord blood exosomes between normal and preeclampsia pregnancies. These proteins may be involved in the etiology of preeclampsia.
\end{abstract}

R. Jia and J.Li contributed equally to this work.

Lizhou Sun, Ph.D.
and Wei De, Ph.D.
Department of Obstetrics and Gynecology, the First Affiliated Hospital of Nanjing

Medical University, 300 Guangzhou Road, Nanjing 210029 (China); and Nanjing Medical University, 140 Hanzhong Road, Nanjing 210029 (China)

E-Mail drsunlizhou@163.com and E-Mail tehliquids@gmail.com 


\section{Cellular Physiology Cell Physiol Biochem 2015;36:2299-2306 \begin{tabular}{l|l|l} 
and Biochemistry & $\begin{array}{l}\text { DOI 10.1159/000430193 } \\
\text { Published online: July 24, } 2015\end{array}$ & $\begin{array}{l}\text { O 2 2015 S. Karger AG, Basel } \\
\text { www.karger.com/cpb }\end{array}$ \\
\cline { 2 - 3 }
\end{tabular}}

Jia et al.: Exosomes Proteomic Profile Between Normal and Preeclampsia Pregnancies

\section{Introduction}

Preeclampsia is a hypertensive disorder of pregnancy, which affects $2-8 \%$ of all pregnancies and remains one of the leading causes of maternal and fetal morbidity and mortality worldwide [1]. Although the etiology of preeclampsia is largely unknown, recent studies suggest that placental-derived exosomes and their biological content (RNAs and protein) contributed to maternal-fetal communication, immune modulation and trophoblast physiology during pregnancy [2-4]. Syncytin proteins incorporated in placenta exosomes show variation from patients with preeclampsia and are important for cell uptake [5].

Exosomes are microvesicle with a size of $40-160 \mathrm{~nm}$ that are released from various cell types including tumor cells, red blood cells, platelets, lymphocytes, and dendritic cells [6]. They have been isolated from biological fluids, including blood plasma, urine and human breast milk [7-9]. Recent study has indicated that exosomes are composed of a lipid bilayer, and contain proteins, mRNA and miRNA [10]. Exosomes have been demonstrated in regulating immune modulation, and increased levels of maternal circulating exosomes is associated with progression of human pregnancy $[4,11]$.

Previous studies have demonstrated that decreased endothelial progenitor cells and ionized calcium levels were found in umbilical cord blood in preeclampsia [12, 13]. There were significant differences in nucleated red blood cell count and blood rheological properties in the umbilical cord blood between healthy women and women with preeclampsia $[14,15]$. These observations could imply that it is possible to identify functional and/or structural differences in the umbilical cord blood with respect to the risk of developing preeclampsia. To date, little is known about umbilical cord blood exosomes during pregnancy. In this study, we compared the proteomic profiling of human umbilical cord blood exosomes between normal and preeclamptic pregnancies using high-resolution LC-MS/MS technologies. We aimed to find potential proteins that are involved in the etiology of preeclampsia.

\section{Materials and Methods}

\section{Ethics statement}

This study was performed with approval from the Medical Ethics Committee of Nanjing Maternal and Child Health Care Hospital (No. [2012]55). Written informed consent was obtained from all patients.

\section{Sample preparation}

All samples and clinical information were collected at the Nanjing Maternal and Child Health Care Hospital affiliated to Nanjing Medical University. Umbilical cord blood samples were collected from the umbilical vein immediately after delivery of fetus during cesarean section (10 cases for PE and 10 cases for control) according to the standard operating procedure. PE was diagnosed in patients with systolic blood pressure (BP) $\geq 150 \mathrm{mmHg}$ or diastolic $\mathrm{BP} \geq 90 \mathrm{mmHg}$ and with proteinuria $\geq 0.3 \mathrm{~g} / \mathrm{d}$ (in a $24 \mathrm{~h}$ harvest) for a period exceeding $4 \mathrm{~h}$ (Table 1). The detailed patient characteristics are presented in Table 1 . All mothers had the same range of age and gestational age.

\section{Exosome purification and analysis}

Exosomes were prepared from the umbilical cord blood. Briefly, umbilical cord blood was centrifuged at 3,000 $\mathrm{g}$ for $15 \mathrm{~min}$ at 4 degree. Supernatants were then centrifuged at 12,000 $\mathrm{g}$ for $30 \mathrm{~min}$ at 4 degree. Then supernatants were filtered through $0.45 \mu \mathrm{m}$ polyvinylidene fluoride (PVDF) membrane, and isolated in a final ultracentrifugation at 100,000 $\mathrm{g}$ for $180 \mathrm{~min}$ at 4 degree. The exosome pellet was resuspended in PBS or lysis buffer. The resulting exosomes were next analyzed with the Nanosight Nano ZS device (Malvern Instruments, Malvern, UK).

Protein digestion, peptide labeling and depuration

Umbilical cord blood exosomes protein extracts $(100 \mu \mathrm{g})$ from normal and PE subjects were digested with trypsin $(1 \mu \mathrm{g} / \mu \mathrm{L})$. Then the mixture was vacuum freeze-dried, and resuspended in tetraethylammonium 


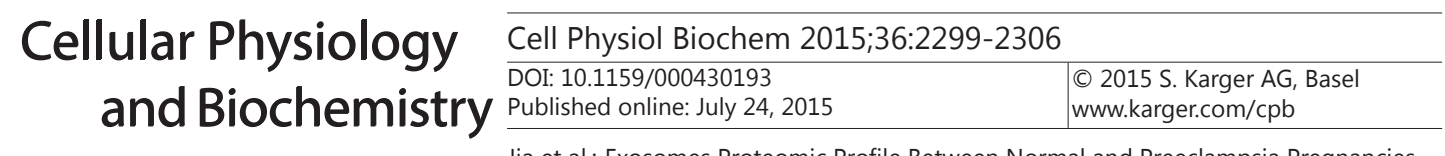

Jia et al.: Exosomes Proteomic Profile Between Normal and Preeclampsia Pregnancies

Table 1. Characteristics of control and PE group. Data are presented as mean \pm SD. ${ }^{* *} P<$ 0.01 compared with control

\begin{tabular}{lll}
\hline & Controls (n=10) & Preeclampsia (n=10) \\
\hline Age (years) & $26.9 \pm 4.8$ & $25.3 \pm 5.3$ \\
Gestational age (weeks) & $38.6 \pm 5.5$ & $34.3 \pm 4.6$ \\
Manner of delivery & Caesarean section & \\
Systolic Blood pressure (mmHg) & $122.3 \pm 7.2$ & $173.2 \pm 10.3^{* *}$ \\
Diastolic Blood pressure (mmHg) & $71.9 \pm 7.4$ & $99.5 \pm 9.2^{* *}$ \\
Proteinuria (g/24h) & 0 & $4.2 \pm 2.5$ \\
Newborn birth weight (g) & $3074.9 \pm 131.3$ & $2705.8 \pm 121.0^{* *}$ \\
Umbilical cord blood volume (ml) & $47.6 \pm 13.5$ & $41.9 \pm 12.8$ \\
\hline
\end{tabular}

bromide (TEAB) containing $0.1 \%$ SDS (water: TEAB=1:1). MALDI TOF/TOF was used to check the digestive efficiency for $1 \mu \mathrm{L}$ of the lysate. 10 cases of $\mathrm{PE}$ or 10 cases of control were randomly divided into 3 groups respectively, indicating the peptide sample of each group was a mixture from 3 or 4 patients. Labeling reagent was then added to the peptides, and isotopic labels of different sizes were used for the different samples. The labeled samples were then dried in vacuo and separated by HPLC and C18 reversed phase chromatography and desalted. The peptides were dissolved by formic acid $(0.1 \%)$.

Mass spectrometry data acquisition

The labeled peptides were analyzed using high-resolution LC-MS (Thermo-fisher Q-Exactive Orbitrap) the same as previously described [16]. Briefly, the MS/MS spectra acquired from precursor ions were submitted to Mascot (version 2.3.01) using the Swissprot Human Library for database search and methionine oxidation for variable modification. The peptide tolerance was set at $15 \mathrm{ppm}, \mathrm{MS} / \mathrm{MS}$ tolerance was set at $20 \mathrm{ppm}$, and the maximum number of missed cleavages was 1 . Meanwhile, qualitative analysis was performed using the median normalization method with the minimum peptides was 1 , the $\mathrm{p}$ value was set at $<0.05$, and the fold change was 1.3 .

Bioinformatics analysis

To further investigate the significance of the differentially expressed proteins, we used SBC Analysis system (Shanghai Biotechnology Corporation, Shanghai, China). Gene ontology (GO) and Kyoto Encyclopedia of Genes and Genomes (KEGG) pathway analyses were applied. Interaction picture of those nine specific proteins was drawn according to Human Protein Reference Database (HPRD) and the Molecular INTeraction database (MINT) databases.

\section{Statistical analysis}

Data were analyzed using SPSS 20.0 software package (SPSS, Chicago, IL, USA) with independentsamples $\mathrm{T}$ test between two groups. All values were represented as mean \pm standard deviation (SD). Statistical significance was defined as $P<0.05$.

\section{Results}

\section{Nanoparticle tracking analysis}

Nanoparticle tracking analysis (NTA) was used to visualize exosomes size and total concentration. By applying the Stokes Einstein equation (Fig. 1A), particle size in the PE group was $120 \pm 37 \mathrm{~nm}$ compared with the control group $(112 \pm 40 \mathrm{~nm})$. For the concentration, there was $32.56 \pm 5.68 \mathrm{E} 8$ particles/ml in the PE group when compared to the control group (27.33 \pm 6.47 E8 particles/ml). A video was taken and the NTA software (Version 2.3, Nano Sight Ltd, Amesbury, UK) tracks the Brownian motion of individual vesicles. A sample video frame shows the static image of exosomes (Fig. 1B).

\section{KARGER}




\section{Cellular Physiology Cell Physiol Biochem 2015;36:2299-2306 and Biochemistry $\begin{array}{ll}\text { DOI: } 10.1159 / 000430193 \\ \text { Published online: July 24, } 2015\end{array}$

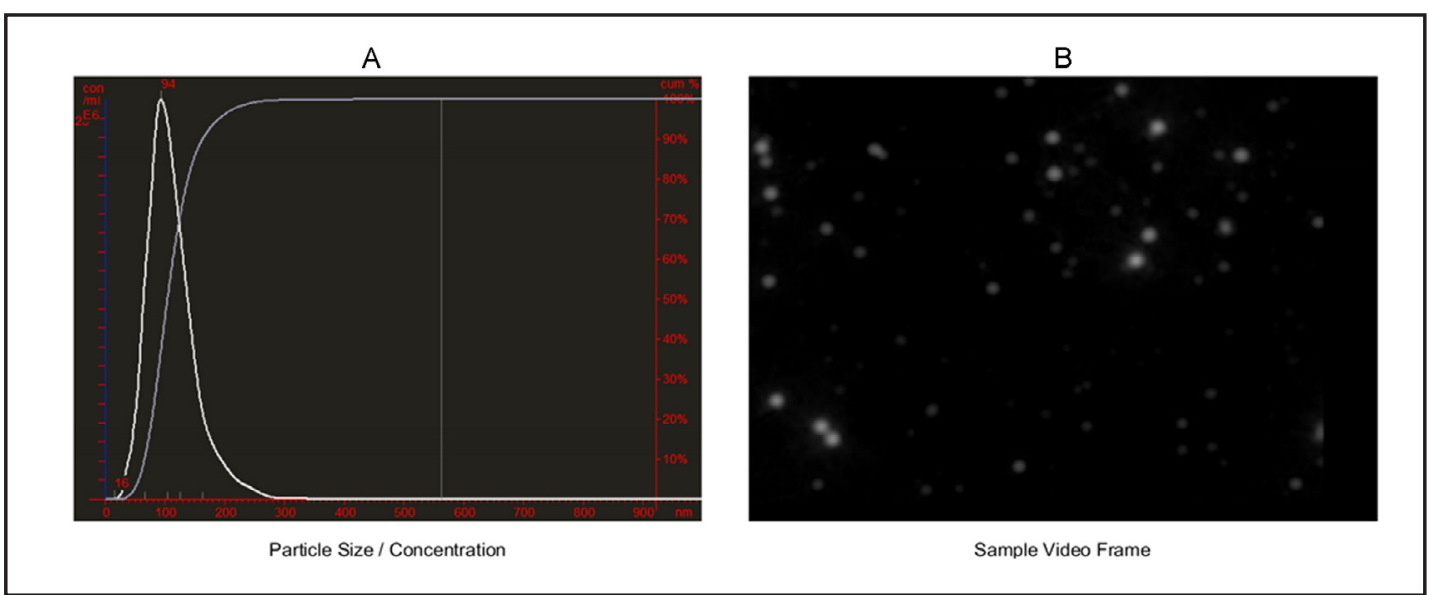

Fig. 1. Representative nanoparticle tracking analysis report. (A) Particle size and concentration analysis using Stokes Einstein equation. (B) A sample video frame shows the static image of exosomes. A white dot points to an exosome.

Table 2. The list of differentially expressed proteins in human umbilical cord blood exosomes from control and preeclampsia pregnancies

\begin{tabular}{|c|c|c|c|}
\hline Protein ID & Protein names & $P$ value & Fold Chang \\
\hline P20851 & C4b-binding protein beta chain(C4BPB) & $3.43 \mathrm{E}-03$ & 2.37 \\
\hline P04003 & C4b-binding protein alpha chain (C4BPA) & $2.02 \mathrm{E}-03$ & 2.27 \\
\hline P07225 & Vitamin K-dependent protein S(PROS1) & $2.36 \mathrm{E}-03$ & 2.18 \\
\hline P00450 & Ceruloplasmin(CP) & $6.77 \mathrm{E}-04$ & 1.90 \\
\hline P01861 & Ig gamma-4 chain $\mathrm{C}$ region(IGHG4) & $1.77 \mathrm{E}-06$ & 1.86 \\
\hline P04196 & Histidine-rich glycoprotein(HRG) & $1.15 \mathrm{E}-03$ & 1.46 \\
\hline B9A064 & Immunoglobulin lambda-like polypeptide 5(IGLL5) & $2.28 \mathrm{E}-05$ & 1.40 \\
\hline H3BM7 4 & NEDD8 ultimate buster 1(NUB1) & $4.17 \mathrm{E}-02$ & 1.39 \\
\hline P01779 & Ig heavy chain V-III region TUR & 3.63E-05 & 1.38 \\
\hline P02776 & Platelet factor $4(\mathrm{PF} 4)$ & $2.76 \mathrm{E}-03$ & 1.37 \\
\hline P01771 & Ig heavy chain V-III region HIL & $2.10 \mathrm{E}-03$ & 1.36 \\
\hline 075382 & Tripartite motif-containing protein 3(TRIM3) & $8.66 \mathrm{E}-04$ & 1.35 \\
\hline P01604 & Ig kappa chain V-I region Kue & $3.24 \mathrm{E}-04$ & 1.35 \\
\hline P06317 & Ig lambda chain V-VI region SUT & $1.62 \mathrm{E}-04$ & 1.31 \\
\hline P02790 & Hemopexin(HPX) & $8.65 \mathrm{E}-03$ & -1.33 \\
\hline P23083 & Ig heavy chain V-I region V35 & $8.70 \mathrm{E}-03$ & -1.37 \\
\hline P04275 & von Willebrand factor(VWF) & $1.01 \mathrm{E}-02$ & -1.39 \\
\hline P05160 & Coagulation factor XIII B chain(F13B) & $4.14 \mathrm{E}-04$ & -1.43 \\
\hline P02766 & Transthyretin(TTR) & $3.86 \mathrm{E}-03$ & -1.52 \\
\hline P02771 & Alpha-fetoprotein(AFP) & $2.36 \mathrm{E}-05$ & -1.57 \\
\hline F5H4W9 & Serum paraoxonase/arylesterase 1(PON1) & $4.27 \mathrm{E}-02$ & -1.77 \\
\hline P02652 & Apolipoprotein A-II(APOA2) & $1.87 \mathrm{E}-02$ & -1.88 \\
\hline J3KNB4 & Cathelicidin antimicrobial peptide(CAMP) & $1.89 \mathrm{E}-02$ & -2.02 \\
\hline P43652 & Afamin(AFM) & $3.89 \mathrm{E}-02$ & -2.05 \\
\hline P02647 & Apolipoprotein A-I(APOA1) & $4.35 \mathrm{E}-02$ & -2.78 \\
\hline P11226 & Mannose-binding protein C(MBL2) & $7.34 \mathrm{E}-04$ & -2.96 \\
\hline P02671 & Fibrinogen alpha chain(FGA) & $4.18 \mathrm{E}-02$ & -3.41 \\
\hline C9JC84 & Fibrinogen gamma chain(FGG) & $4.27 \mathrm{E}-02$ & -3.93 \\
\hline P02675 & Fibrinogen beta chain(FGB) & $3.78 \mathrm{E}-02$ & -4.18 \\
\hline
\end{tabular}

Identification of umbilical cord blood exosomes proteins related to pathological development of preeclampsia

To identify proteins that were differentially expressed in the umbilical cord blood exosomes of normal and PE patients, 221 identified proteins were analyzed on the Thermofisher Q-Exactive Orbitrap. Examination of the mass spectrometry data with Mascot (version 2.3.01) revealed that 29 proteins showed significant (fold change $\geq 1.3, P<0.05$ ) differential expression between the normal and PE patients (Table 2). Compared to the control, 14 
Fig. 2. Chromosome distributions of differentially expressed proteins. Chromosome distributions show the numbers of up and down regulated proteins in different chromosomes.
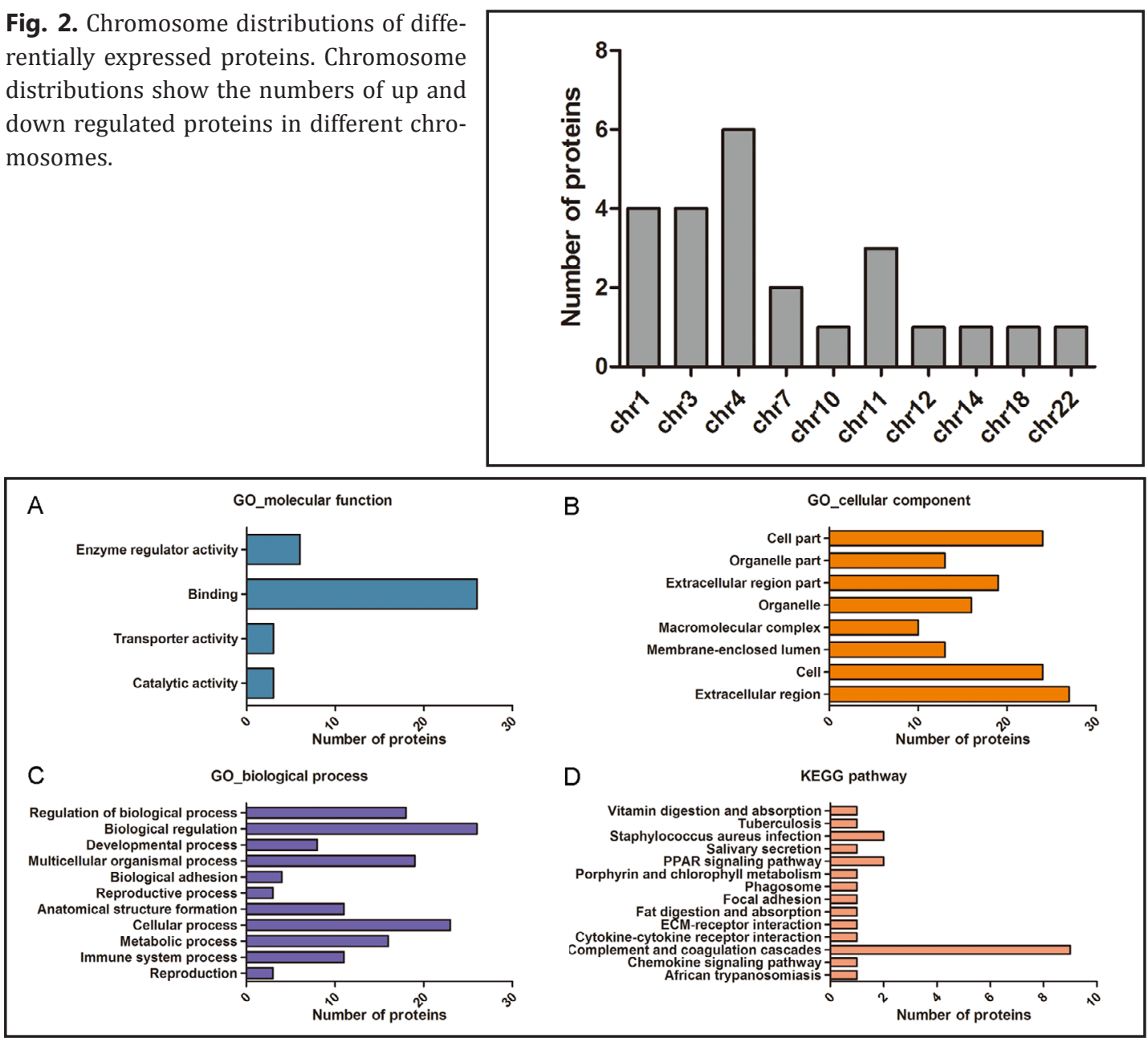

Fig. 3. GO and KEGG pathway analysis. The relative GO terms such as molecular functions (A), cellular component (B) and biological process (C) that associated with the differentially expressed proteins are listed. (D) The relative KEGG pathway that associated with the differentially expressed proteins are listed.

proteins were upregulated and 15 proteins were downregulated in the preeclamptic pregnancies.

Bioinformatics analysis of differentially expressed proteins using SBC Analysis system

To examine the expression signatures of dysregulated proteins, we analyzed upregulated and downregulated proteins according to chromosome distribution. Differentially expressed proteins were located in different chromosomes with most proteins located in chromosome 4 (Fig. 2). GO analysis revealed that these 29 differentially expressed proteins were mainly involved in enzyme regulator activity and binding for the molecular functions (Fig. 3A). The most relevent cellular components for these differentially expressed proteins were extracellular region, cell part and cell (Fig. 3B) that was involved during the pathological changes of PE. For further identification of important biological processes, the results showed that these differentially expressed proteins were significantly involved mostly in biological regulation and cellular process (Fig. 3C). Indeed we found these biological processes are all present in PE development. Furthermore, KEGG pathway analysis indicated that complement and coagulation cascades are mostly associated with PE (Figure 3D). Further analysis identified 9 differentially expressed proteins were related with complement and coagulation cascades (C4BPA, C4BPB, F13B, FGA, FGB, FGG, MBL2, PROS1, VWF; detailed information of these proteins were listed at Table 2). We subsequently analyzed the interaction networks KARGER 


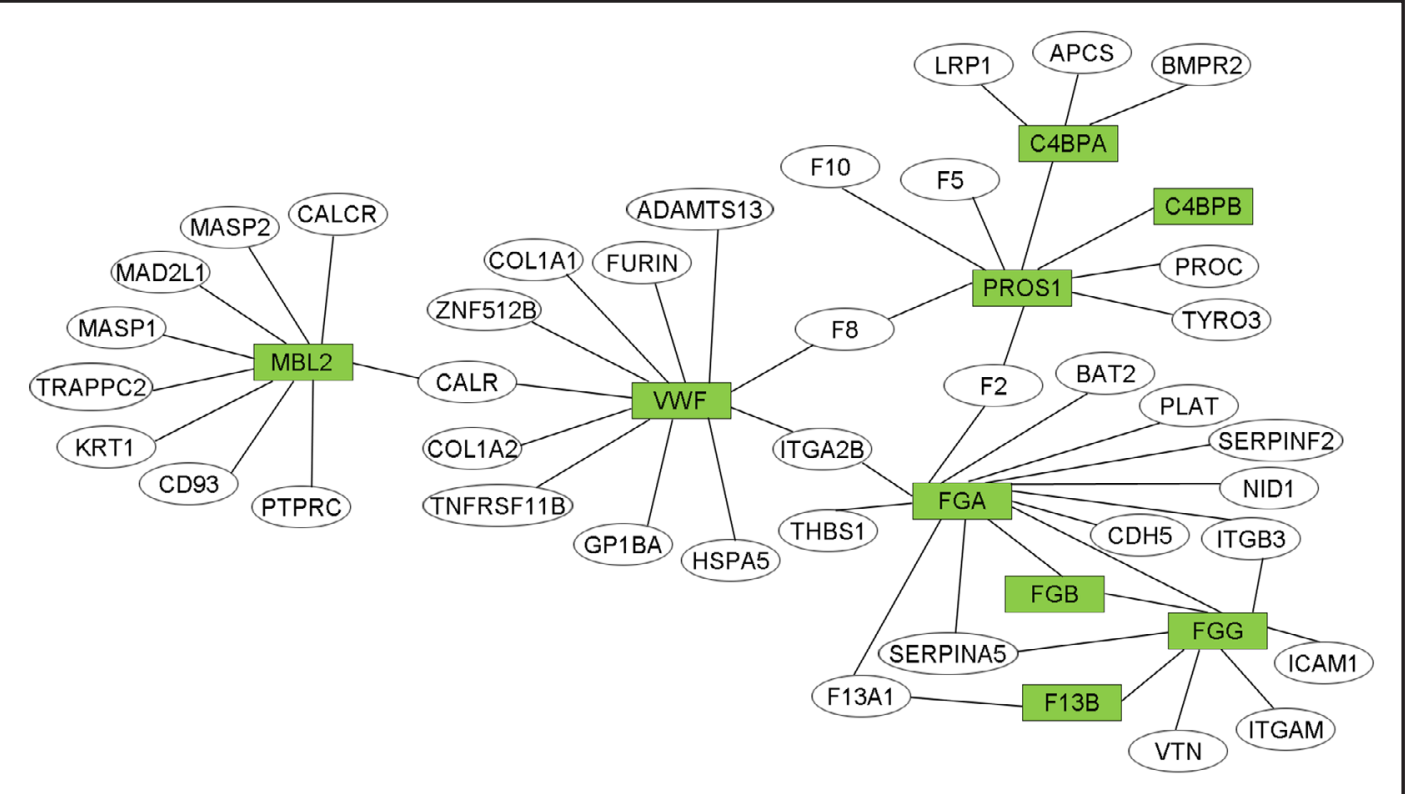

Fig. 4. Interaction networks of these nine proteins related with complement and coagulation cascades. The interaction network was drawn according to Human Protein Reference Database (HPRD) and the Molecular INTeraction database (MINT) databases. Green box indicated these nine proteins. Other proteins are denoted with ellipse.

of these nine proteins according to Human Protein Reference Database (HPRD) and the Molecular INTeraction database (MINT) databases. The results indicated that VWF (von Willebrand factor), PROS1 (vitamin K-dependent protein S) and FGA (Fibrinogen alpha chain) were at the core of interaction networks (Fig. 4).

\section{Discussion}

Preeclampsia (PE) is a specific disorder characterized by the new onset of proteinuria, edema, hypertension and a series of other systematic disorders during pregnancy. A growing body of evidence suggests that placental proteome alterations coordinate the pathological development of PE [17-19]. However, the etiology of PE remains to be elucidated. In this paper, we show that 29 differentially expressed proteins were identified in human umbilical cord blood exosomes between normal and preeclampsia pregnancies with high-resolution mass spectrometry. Importantly, KEGG pathway analysis showed that complement and coagulation cascades are mostly associated with $\mathrm{PE}$, suggesting a possibility that human umbilical cord blood exosomes proteins may be involved in the etiology of preeclampsia via the complement and coagulation cascades. Based on the above and our previous work [20, 21], we obtained a direction for future study on differentially expressed exosomal proteins in umbilical cord blood from PE.

Research on exosomes, most notably in the field of PE, has been increasing over recent years and has demonstrated that these vesicles are involved in cell uptake and placental functions [5, 22]. Recent findings suggest that exosome-associated proteins mediate different exosomal functions, such as miRNA-dependent modulation of gene expression, induced cell signaling and intercellular communication [23-25]. Our study indicated that exosomal proteins from the umbilical cord blood may play crucial roles in the pathogenesis of PE. Furthermore, GO analysis revealed similar information that these differentially expressed exosomal proteins were mainly involved in enzyme regulator activity, binding, extracellular region, cell part, biological regulation and cellular process (Fig. 3). 
Jia et al.: Exosomes Proteomic Profile Between Normal and Preeclampsia Pregnancies

Many proteins in umbilical cord blood have been reported to be associated with PE. Higher soluble Fas ligand levels were identified in umbilical cord blood of PE patients [26]. Large amounts of MMP-9 were found in umbilical cord plasma of preeclamptic subjects [27]. Umbilical cord blood levels of soluble lectin-like oxidized low-density lipoprotein receptor1(sLOX-1) were higher in preeclamptic pregnant [28]. Methemoglobin levels were higher in umbilical cord blood of women PE [29]. Our study characterized 29 differentially expressed proteins in umbilical cord blood exosomes between PE and control samples. Among them, three proteins (VWF, PROS1 and FGA) involved in complement and coagulation cascades were found at the core of interaction network according to KEGG pathway and interaction network analysis (Figure 3 and Figure 4). Previous study reported that elevation in VWF and reduction in its proteolytic enzyme ADAMTS13 activity might have a role in the pathogenesis of PE [30]. FGA has been identified to be serological markers capable of diagnosing PE [31]. In addition, the anticoagulant PROS1 interacting with the complement regulator $\mathrm{C} 4 \mathrm{~b}$-binding protein (C4BP) is a direct physical link between blood coagulation and complement pathways [32]. Our study found that PROS1 was upregulated in PE umbilical cord blood exosome, whereas VWF and FGA were downregulated in PE umbilical cord blood exosome compared with control subjects (Table 2). Those proteins might represent other new mechanisms for PE development during pregnancy. The relevance of those proteins in umbilical cord blood exosomes to PE needs to be further investigated.

\section{Acknowledgments}

This study was supported by grants from the National Natural Science Foundation of China (81200442), Scientific Research Foundation from Department of Health of Jiangsu Province of China (Z201309), Maternal and Child Health Research Foundation of Jiangsu Province of China (F201436), and Nanjing Medical Science and Technique Development Foundation (QRX11209).

\section{Disclosure Statement}

All authors have no conflicts of interest to declare.

\section{References}

1 English F-A, Kenny LC, McCarthy FP: Risk factors and effective management of preeclampsia. Integr Blood Press Control 2015;8:7-12.

2 Ouyang Y, Mouillet JF, Coyne CB, Sadovsky Y: Review: Placenta-specific micrornas in exosomes - good things come in nano-packages. Placenta 2014;35:S69-73.

3 Redman C-W, Sargent IL: Microparticles and immunomodulation in pregnancy and pre-eclampsia. J Reprod Immunol 2007;76:61-67.

4 Taylor D-D, Akyol S, Gercel-Taylor C: Pregnancy-associated exosomes and their modulation of t cell signaling. J Immunol 2006;176:1534-1542.

5 Vargas A, Zhou S, Ethier-Chiasson M, Flipo D, Lafond J, Gilbert C, Barbeau B: Syncytin proteins incorporated in placenta exosomes are important for cell uptake and show variation in abundance in serum exosomes from patients with preeclampsia. FASEB J 2014;28:3703-3719.

6 Cocucci E, Racchetti G, Meldolesi J: Shedding microvesicles: Artefacts no more. Trends Cell Biol 2009;19:4351.

7 Torregrosa P-P, Gutzeit C, Johansson S, Admyre C, Stenius F, Alm J, Scheynius A, Gabrielsson S: Differences in exosome populations in human breast milk in relation to allergic sensitization and lifestyle. Allergy 2014;69:463-471.

8 Caby M-P, Lankar D, Vincendeau-Scherrer C, Raposo G, Bonnerot C: Exosomal-like vesicles are present in human blood plasma. Int Immunol 2005;17:879-887. 


\section{Cellular Physiology Cell Physiol Biochem 2015;36:2299-2306

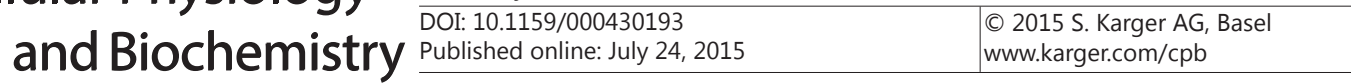

Jia et al.: Exosomes Proteomic Profile Between Normal and Preeclampsia Pregnancies

9 Pisitkun T, Shen RF, Knepper MA: Identification and proteomic profiling of exosomes in human urine. Proc Natl Acad Sci U S A 2004;101:13368-13373.

10 Frydrychowicz M, Kolecka-Bednarczyk A, Madejczyk M, Yasar S, Dworacki G: Exosomes - structure, biogenesis and biological role in non-small-cell lung cancer. Scand J Immunol 2015;81:2-10.

11 Southcombe J, Tannetta D, Redman C, Sargent I: The immunomodulatory role of syncytiotrophoblast microvesicles. PLoS One 2011;6:e20245.

12 Kwon J-Y, Maeng YS, Kwon YG, Kim YH, Kang MH, Park YW: Decreased endothelial progenitor cells in umbilical cord blood in severe preeclampsia. Gynecol Obstet Invest 2007;64:103-108.

13 Yusuf K, Kamaluddeen M, Hasan SU, Al-Awad E, Finch RA, Akierman AR: Ionized calcium levels in umbilical cord blood of women with preeclampsia and normotensive pregnancies. J Matern Fetal Neonatal Med 2012;25:203-205.

14 Csorba R, Yilmaz A, Tsikouras P, Wieg C, Teichmann A, von Tempelhoff GF: Rheological parameters in the umbilical cord blood in moderate and severe forms of preeclampsia. Clin Hemorheol Microcirc 2013;55:391-401.

15 Faraji D-R, Ghanbari A, Asgharnia M, Kian M: Comparison of nucleated red blood cells in the umbilical cord of term neonates in healthy women and women with preeclampsia. Iran J Reprod Med 2013;11:25-30.

16 Miao Z-J, Chen M, Wu H, Ding HJ, Shi ZH: Comparative proteomic profile of the human placenta in normal and fetal growth restriction subjects. Cell Physiol Biochem 2014;34:1701-1710.

17 Wang F-Q Wang L, Shi ZH, Liang GL: Comparative n-glycoproteomic and phosphoproteomic profiling of human placental plasma membrane between normal and preeclampsia pregnancies with high-resolution mass spectrometry. PLoS One 2013;8:e80480.

18 Wang F-Q Shi ZH, Wang P, You W, Liang GL: Comparative proteome profile of human placenta from normal and preeclamptic pregnancies. PLoS One 2013;8:e78025.

19 Shi Z-H, Long W, Zhao C, Guo XR, Shen R, Ding HJ: Comparative proteomics analysis suggests that placental mitochondria are involved in the development of pre-eclampsia. PLoS One 2013;8:e64351.

20 Jia R-Z, Ding GC, Gu CM, Huang T, Rui C, Wang YX, Lu Q: Cdx2 enhances htr-8/svneo trophoblast cell invasion by altering the expression of matrix metalloproteinases. Cell Physiol Biochem 2014;34:628-636.

21 Liu L, Zhang X, Rong C, Rui C, Ji H, Qian YJ, Jia RZ, Sun LZ: Distinct DNA methylomes of human placentas between pre-eclampsia and gestational diabetes mellitus. Cell Physiol Biochem 2014;34:1877-1889.

22 Lokossou A-G, Toudic C, Barbeau B: Implication of human endogenous retrovirus envelope proteins in placental functions. Viruses 2014;6:4609-4627.

23 Fox M-J, Gao H, Smith-Kinnaman WR, Liu Y, Mosley AL: The exosome component rrp6 is required for rna polymerase ii termination at specific targets of the nrd1-nab3 pathway. PLoS Genet 2015;10:e1004999.

24 Yamaguchi T, Izumi Y, Nakamura Y, Yamazaki T, Shiota M, Sano S, Tanaka M, Osada-Oka M, Shimada K, Miura $\mathrm{K}$, Yoshiyama M, Iwao H: Repeated remote ischemic conditioning attenuates left ventricular remodeling via exosome-mediated intercellular communication on chronic heart failure after myocardial infarction. Int J Cardiol 2015;178:239-246.

25 Zhang J, Li S, Li L, Li M, Guo CY, Yao J, Mi SL: Exosome and exosomal microrna: Trafficking, sorting, and function. Genomics Proteomics Bioinformatics 2015;13:17-24.

26 Kuntz T-B, Christensen RD, Stegner J, Duff P, Koenig JM: Fas and fas ligand expression in maternal blood and in umbilical cord blood in preeclampsia. Pediatr Res 2001;50:743-749.

27 Galewska Z, Romanowicz L, Jaworski S, Bankowski E: Gelatinase matrix metalloproteinase (mmp)-2 and mmp-9 of the umbilical cord blood in preeclampsia. Clin Chem Lab Med 2008;46:517-522.

28 Tuten A, Erman H, Korkmaz GG, Oncul M, Gelisgen R, Sozer V, Acikgoz S, Simsek G, Uzun H: Comparison of maternal and umbilical cord blood soluble lectin-like oxidized low-density lipoprotein receptor 1 levels in early- and late-onset preeclampsia. Arch Gynecol Obstet 2014;290:1007-1013.

29 Yusuf K, Wilson RD, Kamaluddeen M, Franta J, Hasan SU, Akierman A: Methemoglobin levels in umbilical cord blood of women with intrauterine growth restriction and preeclampsia. J Matern Fetal Neonatal Med 2014;27:789-794.

30 Aref S, Goda H: Increased vwf antigen levels and decreased adamts13 activity in preeclampsia. Hematology 2013;18:237-241.

31 Wen Q Liu LY, Yang T, Alev C, Wu S, Stevenson DK, Sheng G, Butte AJ, Ling XB: Peptidomic identification of serum peptides diagnosing preeclampsia. PLoS One 2013;8:e65571.

32 Dahlback B: C4b-binding protein: A forgotten factor in thrombosis and hemostasis. Semin Thromb Hemost 2011;37:355-361. 